\title{
TEM Simulations of Tilted Atomically Thin Hexagonal Boron Nitride Sheets
}

\author{
M.L. Odlyzko and K.A. Mkhoyan
}

Department of Chemical Engineering and Materials Science, University of Minnesota, Twin Cities, Minneapolis, MN 55455

Atomically thin hexagonal boron nitride (h-BN), comprised of covalently bonded honeycomb sheets bound by weakly ionic interplanar attractions, has garnered significant interest as a structural analog of graphene with distinctive chemical and electronic properties [1]. TEM characterization is an ideal candidate for atomic-scale analysis of the structure and bonding of few-layer h-BN, and the imaging of h-BN flakes with regions as thin as a single layer has been demonstrated by both bright-field conventional TEM (BF-CTEM) [2,3] and annular-dark-field scanning TEM (ADF-STEM) [4].

Although the BF-CTEM studies have shown that regions of different thickness can be distinguished by relative phase measurements extracted from through-focal-series data, it is useful to study alternative means to determine the thickness of few-layer h-BN. In particular, it is desirable to determine thickness from unprocessed data. To this end, we have employed TEM simulations to demonstrate that sheets of thickness 1 to 4 layers can be clearly distinguished by the evolution of ADF-STEM images and selected-area diffraction patterns (SADPs) as the samples are tilted away from the [0001] zone axis orientation.

Simulations were performed using the TEMSIM multislice package [5]. Images and diffraction patterns were simulated for a $100 \mathrm{keV}$ aberration-corrected microscope. Sheets 1-4 layers thick were analyzed at tilts $0,20,50,100,200$, and $500 \mathrm{mrad}$ relative to [0001] about the $<10-10>$ ("xtilt") and $<11-20>$ ("y-tilt") crystallographic axes. Thermal diffuse scattering was included by averaging many phonon configurations at $300 \mathrm{~K}$.

Similar to the standard study on few-layer graphite [6], parallel-beam diffraction simulations show that SADPs from single-layer h-BN maintain full hexagonal symmetry irrespective of tilt whereas thicker sheets have spots fade and disappear. Figure 1 compares tilt effects on parallel-beam diffraction for x-tilts of 1-layer and 2-layer h-BN. Similar patterns are observed for $y$-tilts.

ADF-STEM images were simulated for a high-angle annular detector with acceptance angle range 54-340 mrad. Both x-tilt (figure 2) and y-tilt (figure 3) series show that 1-layer h-BN only slightly distorts the hexagonal symmetry of the image, while regions 2-4 layers thick exhibit distinctive smearing patterns as the result of the multi-atom (0001) columns being tilted off of the optic axis. This suggests that ADF-STEM imaging also can be used to distinguish 1-layer h-BN from multilayer samples [7].

References

[1] D. Golberg et al., ACS Nano 4 (2010) 2979.

[2] C. Jin et al., Phys. Rev. Lett. 102 (2009) 195505.

[3] N. Alem et al., Phys. Rev. B 80 (2009) 155425.

[4] O.L. Krivanek et al, Nature 464 (2010) 571.

[5] E.J. Kirkland, Advanced Computing in Electron Microscopy, Springer, New York, 2010. 
[6] J.C. Meyer et al., Solid State Comm. 143 (2007) 101.

[7] This research was supported by University of Minnesota and NSF grant DMR-1006706

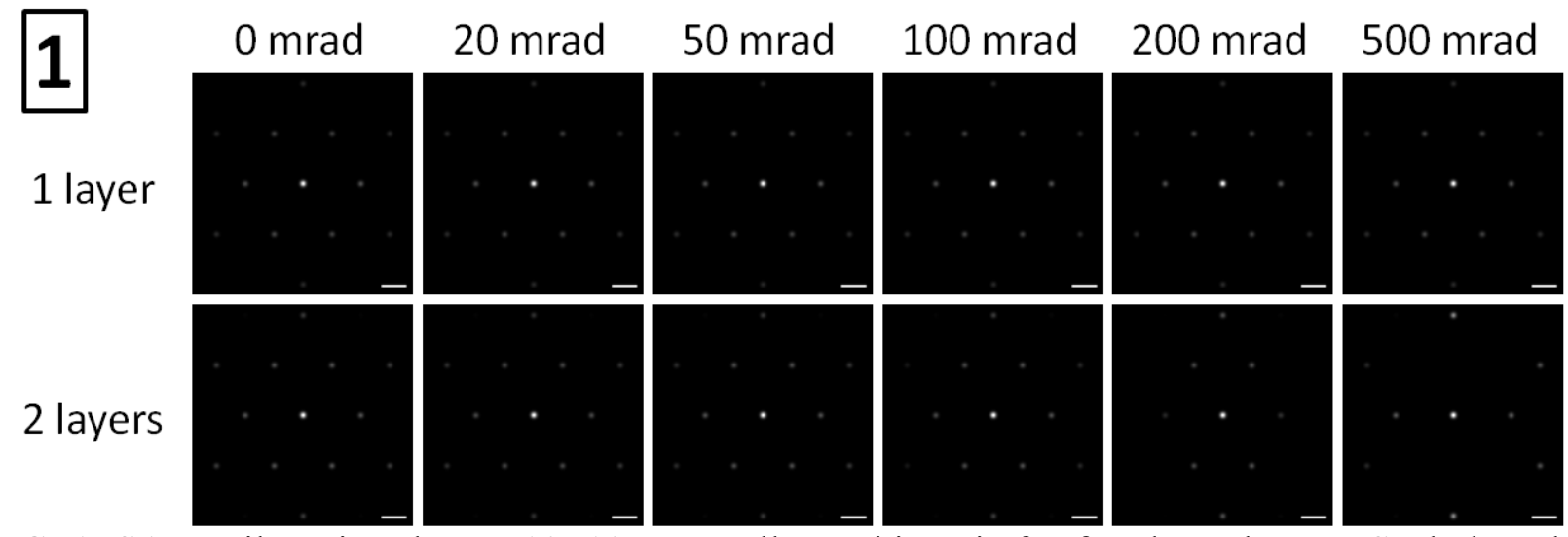

FIG. 1. SADP tilt series about $<10-10>$ crystallographic axis for few-layer $h-B N$. Scale bars have length $2 \mathrm{~nm}^{-1}$.

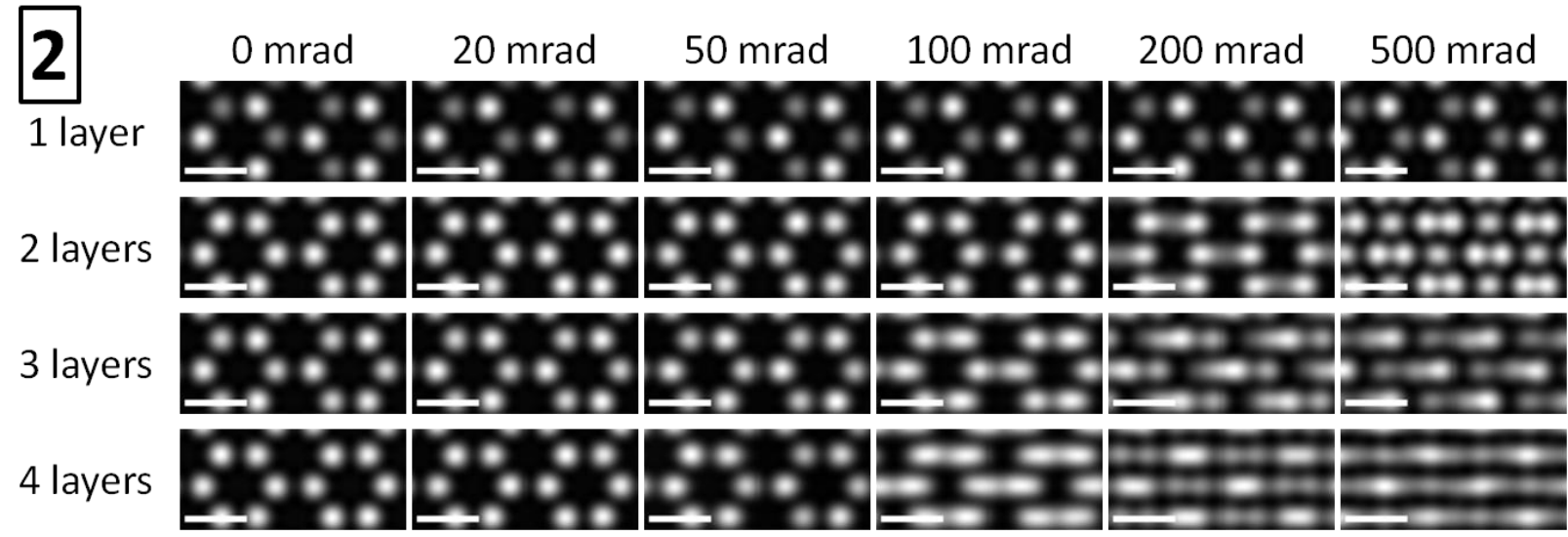

FIG. 2. ADF-STEM image tilt series about $<10-10>$ crystallographic axis for few-layer $h-B N$. Scale bars have length $2 \AA$.

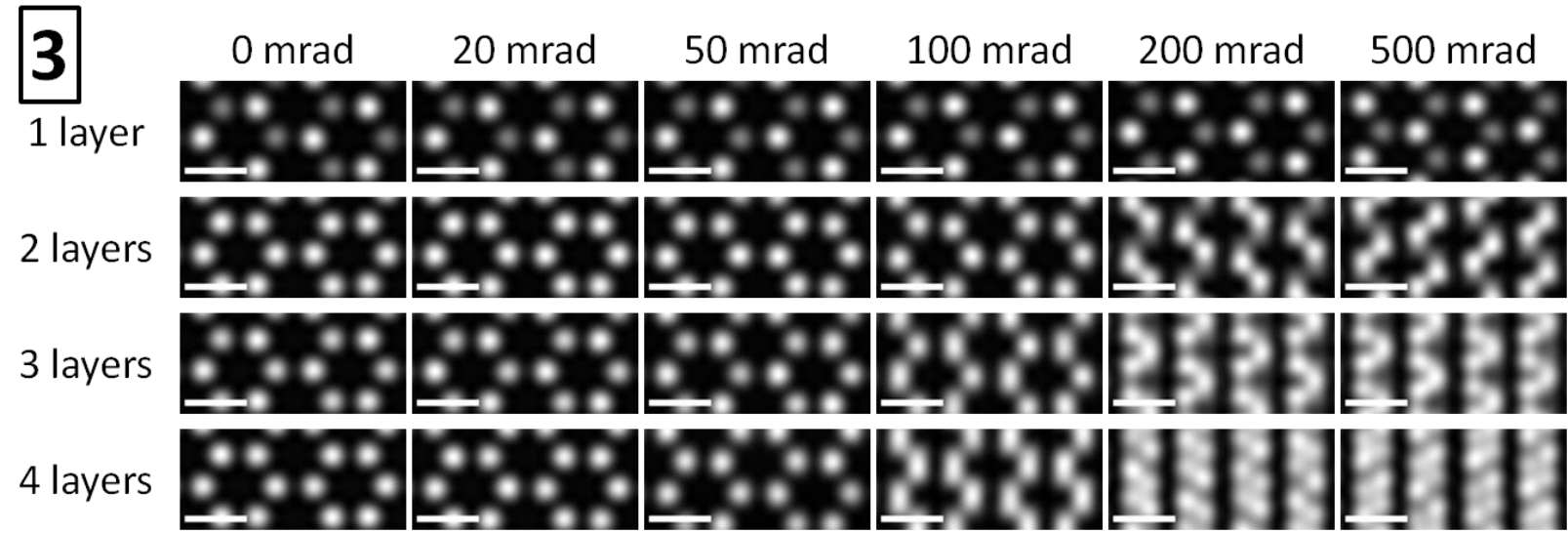

FIG. 3. ADF-STEM image tilt series about $<11-20>$ crystallographic axis for few-layer h-BN. Scale bars have length $2 \AA$. 